\title{
PERTURBATIVE SERIES AND THE MODULI SPACE OF RIEMANN SURFACES
}

\author{
R. C. PENNER
}

\section{Introduction}

In this paper, we use some techniques from quantum field theory to compute quantities related to symmetry groups of pairs $(F, G)$, where $F$ is a surface and $G$ is a spine of $F$. As a consequence of our computation, we derive a certain cohomological invariant of the mapping class group $\mathrm{MC}_{g}^{s}$ of homotopy classes of orientation-preserving homeomorphisms of the genus $g$ surface with $s$ points removed. This invariant, the virtual Euler characteristic $\chi_{v} \mathbf{M C}_{g}^{s}$ of $\mathbf{M C}_{g}^{s}$, is defined as follows. If $A$ is a group which contains a torsion-free subgroup $B$ of finite index and every such subgroup has finitely generated integral homology, then we define

$$
\chi_{v} A=\chi B /[A: B],
$$

where $\chi B$ denotes the usual Euler characteristic of $B$. (It is an exercise to check that $\chi_{v} A$ is independent of the choice of $B$.) We will find that

$$
\chi_{v} \mathrm{MC}_{g}^{s}=(-1)^{s} \frac{(s+2 g-3) !(2 g-1)}{s !(2 g) !} B_{2 g}
$$

for $s \geqslant 1, g \geqslant 0$, and $2 g-2+s>0$, where $B_{2 g}$ denotes the $2 g$ th Bernoulli number.

This result is proved by Harer and Zagier [5] using computational techniques (rediscovered in [5]) which are related to the perturbative series of quantum field theory (see [2] and $\$ 2$ below). Herein, we apply a variant of the full perturbative series machine to capture the equivariant combinatorics of certain cell decompositions of foliated fiber bundles over various Teichmüller spaces. In contrast, [5] begins with cell decompositions of the Teichmüller spaces themselves, and only part of the perturbative series machinery is applicable in

Received January 31, 1986 and, in revised form, December 15, 1986. The author was partially supported by the National Science Foundation. 
this setting. Thus, our treatment of the combinatorics is more direct than that in [5].

$\$ 1$ introduces the category of objects which we will investigate and poses a general counting problem which we solve in subsequent sections. We also recall some work from [6] which explains the connection between our counting problem and the motivating problem of computing $\chi_{v} \mathrm{MC}_{g}^{s}$. $\$ 2$ develops some material which allows us to reformulate our counting problem in terms of integrals over the hermitian matrices; insofar as $\$ 2$ represents an extension of the usual perturbative series/Feynman diagram technique from physics and for completeness, we give a reasonably self-contained account. In $\S 3$, we compute the integrals developed in $\$ 2$ using orthogonal polynomials and generating functions; this is the heart of the paper. The computation is quite delicate and has a life of its own; indeed it is surprising that we obtain a closed form solution to our counting problem as an asymptotic series. It is also noteworthy that the technique of perturbative series from particle physics so effectively captures the combinatorics of the bundle over Teichmüller space described in [6].

It is a pleasure to thank Jürg Fröhlich for introducing me to perturbative series and for his encouragement and enthusiasm; I similarly thank Charlie Epstein for his unflagging interest. Further, I acknowledge useful discussions with Elliot Lieb, Matt Visser, Scott Yost, and Don Zagier.

\section{Fat graphs and Teichmüller space}

By a graph $G$ we mean simply a one-dimensional $\mathrm{CW}$-complex, so that $G$ consists of vertices and open edges. The boundary of an edge may consist of a single vertex, and two distinct edges may have the same vertices. An edge of the first barycentric subdivision of $G$ is called a half-edge of $G$. The valence of a vertex $v$ is the number of half-edges incident on it, and we say $v$ is $k$-valent if this number is $k$. We stipulate throughout this paper that each vertex of $G$ is at least tri-valent.

A fattening of $G$ is a specification of cyclic order on the half-edges of $G$ incident on $v$ for each vertex $v$ of $G$, and a graph together with a fattening is called a fat graph. (The terminology "embedded graph" occurs in the literature.) In practice, we may specify a fat graph by drawing a planar projection of a graph embedded in space: a neighborhood of the vertex set is required to be embedded in the plane, and the collection of half-edges incident on a vertex inherits a cyclic (say counter-clockwise) order from the orientation of the plane; away from the vertices, we introduce under/over crossings. See Figure 1.1 for several examples and notice that the first two examples correspond to 
distinct fattenings of a common underlying graph. It is easy to see that any fat graph may be so represented.

A fat graph $G$ determines an oriented surface $F(G)$ with spine $G \subset F(G)$ (i.e., the inclusion is a homotopy equivalence) as follows. Suppose $G$ is represented by a planar projection and let $N$ denote a regular neighborhood of the vertex set in the plane. $N$ inherits an orientation from that of the plane, and if $e$ is an edge of $G$, then we attach a band to $N$ between the endpoints of $e$ preserving orientation to construct the surface $F(G)$. Several examples are given in Figure 1.2. A marking on $G$ is a marking (in the sense of Riemann surfaces) on $F(G)$.
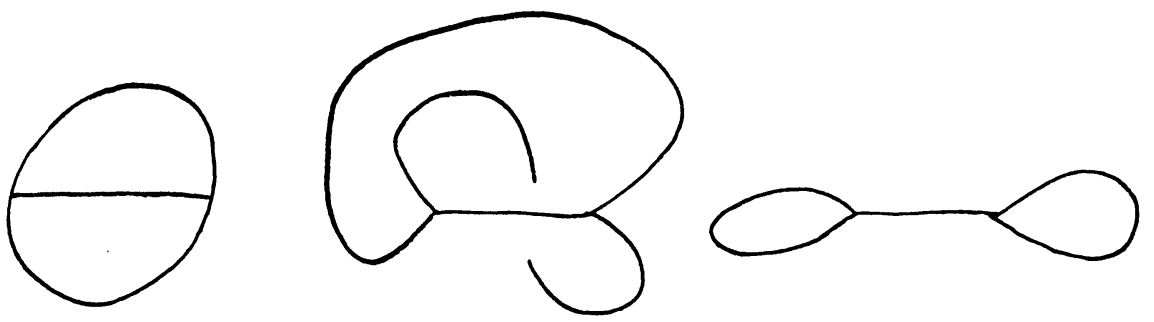

FIGURE 1.1
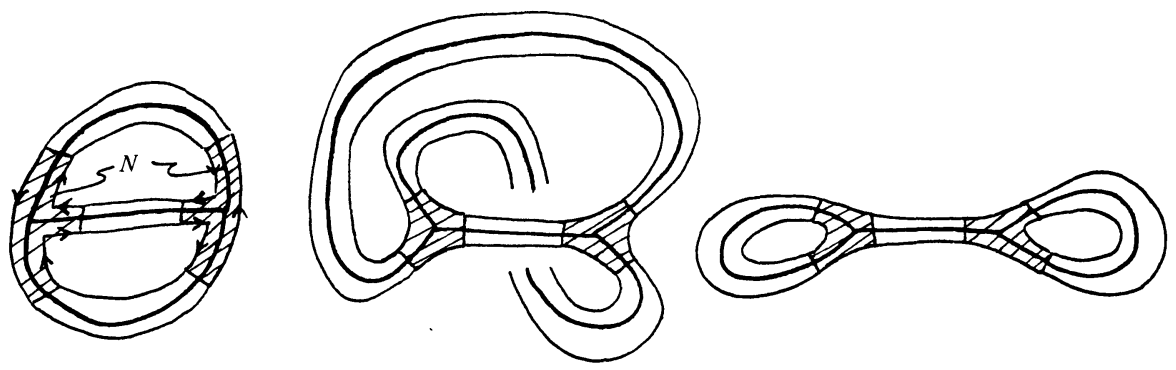

FIGURE 1.2

Suppose that $e$ is an edge of a fat graph with endpoints $w_{1} \neq w_{2}$. We define another fat graph $G^{\prime}$ whose underlying graph arises from that of $G$ by collapsing the edge $e$ and coalescing the vertices $w_{1}$ and $w_{2}$ to form a vertex $w^{\prime}$ of $G^{\prime}$ as in Figure 1.3. The cyclic order on half-edges incident on $w^{\prime}$ is defined in the natural way by combining the cyclic orders about $w_{1}$ and $w_{2}$. We say $G^{\prime}$ arises from $G$ by a Whitehead collapse. Notice that $F(G)$ is homeomorphic to $F\left(G^{\prime}\right)$, and a marking on $G$ induces a marking on $G^{\prime}$. 


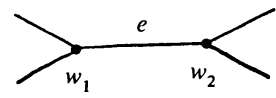

$G$

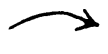

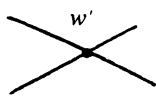

$G^{\prime}$

FIGURE 1.3

A morphism $f: G_{1} \rightarrow G_{2}$ of underlying graphs (i.e., a map on the sets of edges and of vertices preserving the graph structure) is a morphism of fat graphs if $f$ preserves the cyclic ordering on half-edges about each vertex. It is readily seen that a morphism $f: G_{1} \rightarrow G_{2}$ of fat graphs corresponds to a homotopy class of (orientation-preserving) continuous maps of pairs $\left(F\left(G_{1}\right), G_{1}\right) \rightarrow\left(F\left(G_{2}\right), G_{2}\right)$. Two marked fat graphs $F_{g}^{s} \rightarrow F\left(G_{1}\right)$ and $F_{g}^{s} \rightarrow$ $F\left(G_{2}\right)$ are (marked) isomorphic if there is an isomorphism $\left(F\left(G_{1}\right), G_{1}\right) \rightarrow$ $\left(F\left(G_{2}\right), G_{2}\right)$ of fat graphs so that the diagram

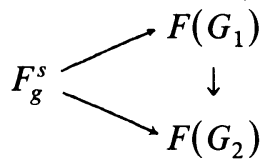

commutes up to homotopy.

Let $[G]$ denote the isomorphism class of the fat graph $G$ and let $\Gamma[G]$ denote the automorphism group. We also define several numerical invariants of fat graphs. Let

$s[G]=\#\{$ boundary components of $F(G)\}$,

$v_{k}[G]=\#\{k$-valent vertices of $G\}$,

so $v_{1} \equiv v_{2} \equiv 0$ by assumption. For instance, the invariants of the fat graphs in Figure 1.1 are $v_{3}=2$ in all three examples with $s=3,1,3$, respectively. Notice that the topological type of $F(G)$ is uniquely determined by these invariants; indeed the Euler characteristic is

$$
\chi(F(G))=\chi(G)=\frac{1}{2} \sum_{k}(2-k) v_{k}[G]
$$

and the genus of $F(G)$ is

$$
g[G]=\frac{1}{4}\left\{4-2 s[G]+\sum_{k}(k-2) v_{k}[G]\right\} .
$$

Finally, we introduce the total valence $V[G]=\sum_{k} v_{k}[G]$ for convenience and remark that

$$
\# \Gamma[G] \leqslant \prod_{k}\left\{v_{k}[G] ! k^{v_{k}[G]}\right\}
$$


In this work, we compute the following generating function for orders of automorphism groups of fat graphs:

$$
\Phi_{c}(I, N)=\sum_{\substack{[G] \text { so that } G \\ \text { is connected } \\ \text { and }-2 \chi(G)=I}} \frac{(-1)^{V[G]} N^{s[G]}}{\# \Gamma[G]} .
$$

The argument $I$ determines the Euler characteristic of contributing fat graphs, and for each $I, \Phi_{c}(I, N)$ is a rational polynomial in $N$. Notice that $\Phi_{c}(I, N)$ $=0$ for $I$ odd. The computation proceeds in two main steps occupying $\$ \S 2$ and 3, respectively. The first step involves the technique of perturbative series from quantum field theory (see [2] for instance), and the second involves a rather delicate computation with generating functions and orthogonal polynomials. In the remainder of this section, we briefly sketch some material from [6], which uncovers the motivation from Teichmüller theory for studying $\Phi_{c}(I, N)$.

Let $\mathrm{MC}_{g}^{s}$ denote the (full) mapping class group (see [3]) of $F_{g}^{s}$ corresponding to isotopy classes of orientation-preserving diffeomorphisms of $F_{g}^{s}$ (which may permute the punctures), and let $\mathscr{T}_{g}^{s}$ denote the Teichmüller space (see [1]) of $F_{g}^{s}$ corresponding to the space of marked conformal classes of complete finite-area metrics of constant curvature -1 on $F_{g}^{s}$. We assume that $2 g-2+s$ $>0$, so that within each marked conformal class, there is a canonical Poincaré metric on $F_{g}^{s}$. We define $\tilde{\mathscr{T}}_{g}^{s}$ to be the Cartesian product of $\mathscr{T}_{g}^{s}$ and the space of horocycles (for the Poincaré metric) on $F_{g}^{s}$, one horocycle about each puncture. The "decorated Teichmüller space" $\tilde{\mathscr{T}}_{g}^{s}$ is homeomorphic to $\mathscr{T}_{g}^{s} \times$ $\mathbf{R}_{+}^{s}$ (where $\mathbf{R}_{+}=\{t \in \mathbf{R}: t>0\}$ ), and $\mathrm{MC}_{g}^{s}$ acts on $\tilde{\mathscr{T}}_{g}^{s}$ by the action on horocycles of the representative diffeomorphism which respects the Poincare metrics. The fibration $\tilde{\mathscr{T}}_{g}^{s} \rightarrow \mathscr{T}_{g}^{s}$ is mapping class group equivariant, and there is a canonical section.

Let $\mathbf{M}$ denote Minkowski three-space, so that $\mathbf{M}$ is a real vector space of dimension three with a bilinear pairing $\langle\cdot, \cdot\rangle$ which admits a basis $\left(e_{0}, e_{1}, e_{2}\right)$ so that $\left\langle e_{i}, e_{j}\right\rangle=0$ if $i \neq j$ and $-\left\langle e_{0}, e_{0}\right\rangle=\left\langle e_{1}, e_{1}\right\rangle=\left\langle e_{2}, e_{2}\right\rangle=1$. The sheet

$$
\mathbf{H}=\left\{v \in \mathbf{M}:\langle v, v\rangle=-1 \text { and }\left\langle v, e_{0}\right\rangle<0\right\}
$$

of the hyperboloid inherits a Riemannian metric from the pairing and gives a model for the hyperbolic plane. We homeomorphically identify the positive light-cone

$$
L^{+}=\left\{v \in \mathbf{M}:\langle v, v\rangle=0 \text { and }\left\langle e, e_{0}\right\rangle<0\right\}
$$


with the space of horocycles in the hyperbolic plane (with the geometric topology) by letting $w \in L^{+}$correspond to the horocycle $\{v \in \mathbf{H}:\langle w, v\rangle=$ $-1\}$. The group of linear isomorphisms of $\mathbf{M}$ preserving the form, the orientation on $\mathbf{M}$, and the sheet $\mathbf{H}$ is denoted $\mathrm{SO}^{+}(1,2)$; this group corresponds to the Möbius group of orientation-preserving isometries of the hyperbolic plane.

A point of $\mathscr{T}_{g}^{s}$ gives rise to an isomorphism

$$
\pi_{1} F_{g}^{s} \rightarrow \Gamma<\mathrm{SO}^{+}(1,2),
$$

where $\Gamma$ is a discrete group defined up to conjugacy in $\mathrm{SO}^{+}(1,2)$ and the point in ${\mathscr{T}_{g}}^{s}$ is represented by $\mathbf{H} / \Gamma$. A specification of horocycles on $F_{g}^{s}$ gives rise to a $\Gamma$-invariant set $\mathscr{B} \subset L^{+}$via the correspondence above between $L^{+}$and the space of horocycles. (Explicitly, choose a parabolic $\gamma_{i} \in \Gamma$ corresponding to the $i$ th puncture, let $z_{i} \in L^{+}$be fixed by $\gamma_{i}$ and correspond to the chosen horocycle about the $i$ th puncture, $i=1, \cdots, s$, and let $\mathscr{B}=\bigcup_{i} \Gamma z_{i}$.) Since the horocycles may be chosen to be disjointly embedded in $F_{g}^{s}$, the origin in $\mathbf{M}$ is never an accumulation point of $\mathscr{B}$; since the set of eigenvectors (rays) of hyperbolic elements of $\Gamma$ is dense in $L^{+}$, it follows that $\mathscr{B}$ is discrete in $L^{+}$ itself. Thus, a point $\tilde{\Gamma} \in \tilde{\mathscr{T}}_{g}^{s}$ gives rise to a discrete group $\Gamma<\operatorname{SO}^{+}(1,2)$ and a $\Gamma$-invariant discrete set $\mathscr{B} \subset L$. Let $K$ be the euclidean closed convex hull of $\mathscr{B}$ in $\mathbf{M}$; since $\Gamma$ acts linearly, $K$ is a $\Gamma$-invariant convex body. A pair of distinct points in $L^{+}$gives rise to an arc in $F_{g}^{s}$ connecting punctures: the plane in $\mathbf{M}$ through the origin containing the pair of points intersects $\mathbf{H}$ in a geodesic which projects to such an arc in $F_{g}^{s}=\mathbf{H} / \Gamma$. It turns out that the edges of $\partial K$ inside $L^{+}$correspond to a disjointly embedded (finite) family $\Delta(\tilde{\Gamma})$ of such arcs in $F_{g}^{s}$, none of which is homotopic into a puncture and no two of which are parallel, so that components of $F_{g}^{s}-\Delta(\tilde{\Gamma})$ are contractible (Theorem 4.5 of $[6])$. We regard $\Delta(\tilde{\Gamma})$ as defined only up to isotopy and call such a decomposition of $F_{g}^{s}$ an "ideal cell decomposition."

Having associated $\Delta(\tilde{\Gamma})$ to $\tilde{\Gamma} \in \tilde{\mathscr{T}}_{g}^{s}$, consider the decomposition $\mathscr{C}$ of $\tilde{\mathscr{T}}_{g}^{s}$ consisting of all

$$
C(\Delta)=\left\{\tilde{\Gamma} \in \tilde{\mathscr{T}}_{g}^{s}: \Delta(\tilde{\Gamma}) \subset \Delta\right\}
$$

as $\Delta$ ranges over the ideal cell decomposition of $F_{g}^{s}$. One of the main results from [6] (Theorem 5.5) is that $\mathscr{C}$ is an $\mathrm{MC}_{g}^{s}$-invariant cell decomposition of $\tilde{\mathscr{T}}_{g}^{s}$ itself. Moreover, the isotropy group of $C(\Delta)$ in $\mathrm{MC}_{g}^{s}$ is isomorphic to the topological symmetry group of the pair $\left(F_{g}^{s}, \Delta\right)$.

Now, the dual of an ideal cell decomposition of $F_{g}^{s}$ is a spine of $F_{g}^{s}$, which corresponds to some marked fat graph $G$ with $F(G)=F_{g}^{s}$; moreover, all such marked fat graphs arise in this way. Notice that if marked fat graphs $G$ and $G^{\prime}$ correspond to ideal cell compositions $\Delta \subset \Delta^{\prime}$ of $F_{g}^{s}$, respectively, then $G$ arises 
from $G^{\prime}$ by Whitehead collapses. The dual of the decomposition $\mathscr{C}$ of $\tilde{\mathscr{T}}_{g}^{s}$ itself is an $\mathrm{MC}_{g}^{s}$-equivariant spine $\Sigma_{g}^{s}$, and $\Sigma_{g}^{s}$ is contractible since $\tilde{\mathscr{T}}_{g}^{s}$ is. Furthermore, the $\mathrm{MC}_{g}^{s}$-stabilizer of a cell in $\Sigma_{g}^{s}$ is isomorphic to the automorphism group of the corresponding fat graph. $\Sigma_{g}^{s}$ is $\mathrm{MC}_{g}^{s}$-equivariantly isomorphic to the fat graph complex $\mathscr{G}_{g}^{s}$ defined as follows. $\mathscr{G}_{g}^{s}$ has one cell for each isomorphism class of marked fat graph $[G]$ with $G$ homotopy equivalent to $F_{g}^{s}$, and the dimension of the cell corresponding to $[G]$ is $\sum_{k}(k-3) v_{k}[G]$. The cell for $[G]$ is a face of the cell for $\left[G^{\prime}\right]$ if $G^{\prime}$ arises from $G$ by Whitehead collapses. $\mathrm{MC}_{g}^{s}$ acts on $\mathscr{G}_{g}^{s}$ by change of marking.

Theorem 1.1. We have the equality

$$
\Phi_{c}(I, N)=\sum_{\substack{g \geqslant 0, s \geqslant 1 \text { so } \\ \text { that } I=4 g-4+2 s}} \chi_{v} \mathrm{MC}_{g}^{(I-4 g+4) / 2} N^{(I-4 g+4) / 2} .
$$

Proof. It follows from the remarks above that $\mathrm{MC}_{g}^{s}$ acts cellularly on $\Sigma_{g}^{s}$ with finite isotropy groups, and $\mathscr{G}_{g}^{s}$ is contractible. Since $\mathrm{MC}_{g}^{s}$ is virtually torsion free, Proposition 4 of [4] therefore asserts that

$$
\chi_{v} \mathrm{MC}_{g}^{s}=\sum_{i, p} \frac{(-1)^{p}}{\# \Gamma\left(\sigma_{i}^{p}\right)}
$$

where $\sigma_{i}^{p}, i=1, \cdots, u_{p}$, is an enumeration of certain of the $p$-dimensional cells of $\mathscr{G}_{g}^{s}$, one representative cell for each $\mathrm{MC}_{g}^{s}$-orbit, and $\Gamma\left(\sigma_{i}^{p}\right)$ denotes the isotropy subgroup of $\sigma_{i}^{p}$ in $\mathrm{MC}_{g}^{s}$. Since the isotropy group of $\sigma_{i}^{p}$ is isomorphic to the fat graph automorphism group of the corresponding fat graph, $\sum_{k}(k-3) v_{k}[G] \equiv V[G] \bmod 2$, and $I=-2 \chi G=4 g-4+2 s$, the result follows. q.e.d.

This theorem gives a motivation for the study of the functions $\Phi_{c}(I, N)$.

\section{Perturbative series}

Consider the vector space $\mathscr{H}^{N}$ of $N$ by $N$ hermitian matrices which supports the adjoint representation of the $N$ by $N$ unitary matrices $\mathscr{U}^{N}$, where

$$
U: H \rightarrow U H U^{-1}, \quad U \in \mathscr{U}^{N}, H \in \mathscr{U}^{N} .
$$

We recall that any $H \in \mathscr{H}^{N}$ is $\mathscr{U}^{N}$-equivalent to a diagonal matrix, and $\mathscr{H}^{N}$ supports the $\mathscr{U}^{N}$-invariant product

$$
d M(H)=\prod_{i=1}^{N} d H_{i i} \prod_{i<j}\left(d \operatorname{Re} H_{i j}\right)\left(d \operatorname{Im} H_{i j}\right)
$$

of Lebesgue measures, where $H=\left(H_{i j}\right) \in \mathscr{H}^{N}$. 
Suppose $v_{3}, \cdots, v_{K}$ is a sequence of nonnegative integers, and set $0=v_{1}=$ $v_{2}=v_{K+1}=v_{K+2}=\cdots$ for convenience. We define

$$
P_{v_{3}, \cdots, v_{K}}(N)=\frac{1}{\mu_{N} \prod_{k} v_{k} !} \int_{\mathscr{H}^{N}} \prod_{k=1}^{K}\left[\frac{\operatorname{tr} H^{k}}{k}\right]^{v_{k}} e^{-\operatorname{tr} H^{2} / 2} d M(H),
$$

where $\operatorname{tr}$ denotes the trace and $\mu_{N}=2^{N / 2} \pi^{N^{2} / 2}$. Notice that $P_{v_{3}, \cdots, v_{K}}(N)=0$ unless $\sum_{k} k v_{k}$ is even. Our main result for this section is

Theorem 2.1. $\quad P_{v_{3}, \cdots, v_{K}}(N)$ is a polynomial in $N$ with no constant term. In fact, we have the equality

$$
P_{v_{3}, \cdots, v_{K}}(N)=\sum_{\begin{array}{c}
{[G] \text { so that }} \\
v_{k}[G]=v_{k} \\
\text { for } k=1,2, \cdots
\end{array}} \frac{N^{s[G]}}{\# \Gamma[G]} .
$$

We emphasize that the sum extends over all such fat graph classes, not necessarily connected.

Proof. Adopting the convention that all indices $k$ run from 1 to $K$ and all indices $i$ (possibly with sub- and superscripts) from 1 to $N$, we have

$$
\begin{aligned}
P_{v_{3}, \cdots, v_{K}}(N) & \frac{1}{\mu_{N} \prod_{k} v_{k} !} \int_{\mathscr{H}^{N}} d M(H) e^{-\operatorname{tr} H^{2} / 2} \prod_{k}\left[\frac{1}{k} \sum_{i_{i}, \cdots, i_{k}} H_{i_{1} i_{2}} H_{i_{2} i_{3}} \cdots H_{i_{k} i_{1}}\right]^{v_{k}} \\
= & \frac{1}{\mu_{N} \prod_{k}\left(v_{k} ! k^{v_{k}}\right)} \int_{\mathscr{H}^{N}} d M(H) e^{-\operatorname{tr} H^{2} / 2} \\
& \cdot \prod_{k} \sum_{\left\{\left\{i_{\nu=1}^{\mu}\right\}: \mu=1, \cdots, v_{k}\right.} \prod_{j=1}^{v_{k}} H_{i_{1}^{j} i_{2}^{j}} H_{i_{2}^{j} i_{3}^{j}} \cdots H_{i_{k}^{j} j_{1}^{j}} \\
= & \frac{1}{\mu_{N} \prod_{k}\left(v_{k} ! k^{v_{k}}\right)} \int_{\mathscr{H}^{N}} d M(H) e^{-\operatorname{tr} H^{2} / 2} \sum_{(*)} \prod_{j=1}^{v_{k}} H_{i_{1 k}^{j} i_{2 k}^{j}} H_{i_{2 k}^{j} i_{3 k}^{j}} \cdots H_{i_{k k}^{j} i_{1 k}^{j},},
\end{aligned}
$$

where $(*)$ denotes the collection of multi-indices,

$$
(*)=\left\{\tau=\left\{i_{\nu k}^{\mu}\right\}: k=1, \cdots, K, \nu=1, \cdots, K, \mu=1, \cdots, v_{k}\right\},
$$

with each $i_{\nu k}^{\mu}$ running from 1 to $N$. The first equality follows from the definition of trace, the second upon taking $v_{k}$ th powers, and the third from pulling the product past the sum.

We will call a collection of indices of the form

$$
\boldsymbol{\sigma}=\left(i_{1 k}^{j}, i_{2 k}^{j}, \cdots, i_{k k}^{j}\right)
$$


occurring in a term of $(*)$ a "cycle of length $k$," so each term in $(*)$ consists of $v_{k}$ cycles of length $k$ for $k=1, \cdots, K$. Such a cycle contributes a factor

$$
H_{i j_{k} i j_{k}} H_{i j_{k} i_{j k}} \cdots H_{i k_{k} i j_{k}}
$$

to the integrand, and we associate the set

$$
S(\sigma)=\left\{\left(i_{1 k}^{j}, i_{2 k}^{j}\right),\left(i_{2 k}^{j}, i_{3 k}^{j}\right), \cdots,\left(i_{k k}^{j}, i_{1 k}^{j}\right)\right\}
$$

of pairs of indices to each cycle $\sigma$.

We introduce the notation

$$
\langle\cdot\rangle=\frac{1}{\mu_{N}} \int_{\mathscr{H}^{N}} d M \cdot e^{-\operatorname{tr} H^{2} / 2}
$$

for the Gaussian moment of '; remark that $\langle 1\rangle=1$ (see Lemmas 3.2 and 3.3 below), and recall the following fundamental result.

Wick's Lemma (see $[2, \S 2]) .\left\langle H_{i_{1} j_{1}} H_{i_{2} j_{2}} \cdots H_{i_{n} j_{n}}\right\rangle$ vanishes for $n$ odd and equals

$$
\sum_{\substack{\text { partitions of } \\\{1, \cdots, n\} \\ n / 2 \text { doubletons }}} \prod_{\substack{\text { partition } \\ \text { elements }\{\alpha, \beta\}}}\left\langle H_{i_{\alpha} j_{\alpha}} H_{i_{\beta} j_{\beta}}\right\rangle
$$

for $n$ even. Furthermore, $\left\langle H_{a b} H_{c d}\right\rangle=\delta_{a d} \delta_{b c}$.

Using this notation, we may write

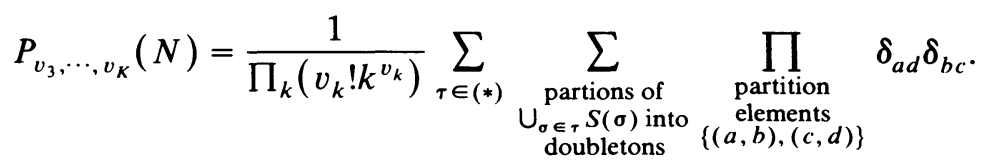

Each term in the sum corresponding to $\tau \in(*)$ and a partition $\mathscr{P}$ of $\bigcup_{\sigma \in \tau} S(\sigma)$ into doubletons contributions either 0 or 1 ; let $x_{\tau}(\mathscr{P})$ denote this contribution.

We wish to associate a fat graph (together with some extra structure) to each term $(\tau, \mathscr{P})$ in the sum. Begin with $v_{k}$ copies of a standard neighborhood in the plane of a $k$-valent vertex; label the vertices $1, \cdots, v_{k}$ for $k=3, \cdots, K$. The boundary of each such neighborhood is naturally decomposed in $k$ "sectors" and $k$ "stubs," which we linearly order by labelling $1,2, \cdots, k$ (see Figure 2.1(a)). Given a cycle $\sigma_{j}=\left(i_{1 k}^{j}, i_{2 k}^{j}, \cdots, i_{k k}^{j}\right)$ of length $k$ in $\tau$, we assign the integers $i_{1 k}^{j}, \cdots, i_{k k}^{j} \in\{1, \cdots, N\}$ to the sectors about the $j$ th vertex in order; we then assign the labels of adjacent sectors to each stub. Furthermore, the orientation of the plane induces an orientation on the neighborhoods so that to each stub is associated one "outgoing" index and one "incoming" index (see Figure 2.1(b)). The cycle of indices $\sigma_{j}$ thus induces a labelling on the stubs about the $j$ th vertex, where each stub is labelled by an ordered pair of integers from $\{1, \cdots, N\}$. 


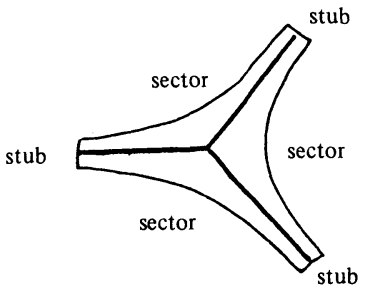

(a)

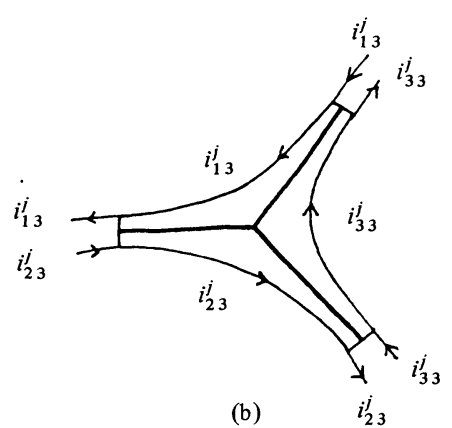

(b)

FigURE 2.1

Now, if an element of $\mathscr{P}$ consists of a pair of elements of $\bigcup_{\sigma \in \tau} S(\sigma)$, then we attach an orientation-preserving band between the corresponding stubs. Doing this for each pair (of pairs) in $\mathscr{P}$ yields a fat graph $G(\mathscr{P})$. Moreover, the $m$ th sector about the $j$ th $k$-valent vertex is labelled with the integer $i_{m k}^{j}$. A fat graph with an assignment of integers from $1, \cdots, N$ to the sectors about each vertex together with a linear ordering on the $k$-valent vertices, $k=$ $3, \cdots, K$, and a linear ordering on the sectors about each vertex is said to be $N$-labelled, so that $G(\mathscr{P})$ is 1-labelled by construction and inherits an $N$ labelling from $\tau$.

Since $\left\langle H_{a b} H_{c d}\right\rangle=\delta_{a d} \delta_{b c}$, the $N$-labelling on $G(\mathscr{P})$ must be constant on sectors corresponding to the same boundary component of $F[G(\mathscr{P})]$ in order for $x_{\tau}(\mathscr{P}) \neq 0$. Thus, we find that

$$
\begin{aligned}
P_{v_{3}, \cdots, v_{K}}(N) & =\frac{1}{\Pi_{k}\left(v_{k} ! k^{v_{k}}\right)} \sum_{\tau \in(*)} \sum_{\text {partitions } \mathscr{P}} x_{\tau}(\mathscr{P}) \\
& =\frac{1}{\Pi_{k}\left(v_{k} ! k^{v_{k}}\right)} \sum_{\text {partitions } \mathscr{P}} N^{s[G(\mathscr{P})]} .
\end{aligned}
$$

Let us be quite explicit about the index set in this last sum. For fixed $v_{3}, \cdots, v_{k}$, we arrange $v_{k}$ "standard" $k$-valent vertices in the plane in a "standard" way, so that there is an induced 1-labelling on any fat graph with this vertex set. An element of the index set is a partition $\mathscr{P}$ into doubletons of the half-edges emanating from these vertices, which in turn specifies a fat graph $G(\mathscr{P})$. There are thus

$$
\left(\sum_{k} k v_{k}-1\right) ! !=\left(\sum_{k} k v_{k}-1\right)\left(\sum_{k} k v_{k}-3\right) \cdots(3)(1)
$$

terms in this sum. By way of example, we enumerate the 15 1-labelled fat graphs in the index set in case $v_{3}=2$ in Figure 2.2. 

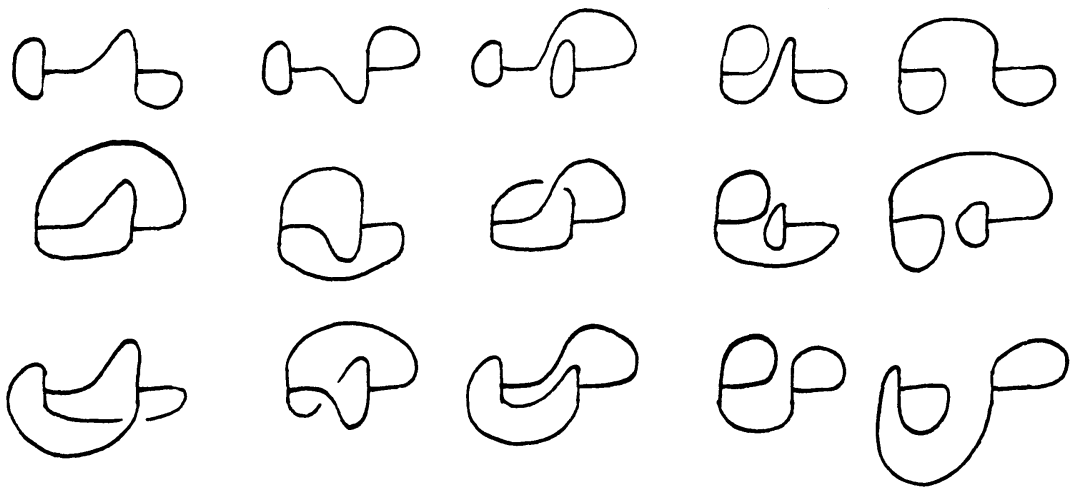

Figure 2.2

Let $\rho[G]$ be the number of appearances of the isomorphism class $[G]$ of unlabelled fat graphs in this index set. For instance, the three fat graph classes with $v_{3}=2$ illustrated in Figure 1.1 have $\rho[G]=3,3,9$ and $\# \Gamma[G]=6,6,2$, respectively. $\Gamma[G]$ acts without fixed points on the set of 1-labellings on the fat graph $G$, and, even though the action is not transitive, each orbit type occurs once in our index set. Evidently, we have

$$
\begin{aligned}
\rho[G]( & \# \Gamma[G])=\prod_{k}\left(v_{k} ! k^{v_{k}}\right) \\
& =\#\left\{1 \text {-labellings on a fat graph } G \text { with } v_{k}[G]=v_{k}, k=1, \cdots, K\right\},
\end{aligned}
$$

and it follows that

$$
\begin{aligned}
P_{v_{3}, \cdots, v_{k}}(N) & =\frac{1}{\prod_{k}\left(v_{k} ! k^{v_{k}}\right)} \sum_{\begin{array}{c}
{[G] \text { with }} \\
v_{k}[G]=v_{k}, \\
k=1,2, \cdots
\end{array}} \rho[G] N^{s[G]} \\
& =\sum_{\substack{[G] \text { with } \\
v_{k}[G]=v_{k} \\
k=1,2, \cdots}} \frac{N^{s[G]}}{\# \Gamma[G]}
\end{aligned}
$$

as desired.

\section{The asymptotic series}

Define $\Phi(I, N)$ exactly as we defined $\Phi_{c}(I, N)$ in $\S 1$ but summing over all (not necessarily connected) fat graph classes with $-2 \chi(G)=I$. Theorem 2.1 has the following immediate consequence. 
Corollary 3.1. We have the equalities

$$
\begin{aligned}
\Phi(I, N) & =\sum_{\Sigma(k-2) v_{k}=I}(-1)^{\Sigma_{k} v_{k}} P_{v_{3}, \cdots, v_{K}}(N) \\
& =\sum_{\Sigma(k-2) v_{k}=I} \frac{1}{\mu_{N} \prod_{k} v_{k} !} \int_{\mathscr{H}^{N}} d M(H) \prod_{k}\left[-\frac{\operatorname{tr} H^{k}}{k}\right]^{v_{k}} e^{-\operatorname{tr} H^{2} / 2} .
\end{aligned}
$$

The index set " $\Sigma(k-2) v_{k}=I$ " means that we sum over all tuples $\left\{v_{k}\right\}_{1}^{K}$ of nonnegative integers with $\Sigma_{k}(k-2) v_{k}=I$ and $v_{1}=v_{2}=0$. It is our intent to numerically evaluate these integrals in closed form by developing a generating function in a nonnegative real variable $t$, which converges for $t>0$ and whose asymptotic series at 0 has coefficients given by $\Phi(I, N)$.

In preparation for this approach, we begin by manipulating the expression $\Phi(I, N)$ itself and recall

Lemma 3.2 (see Appendix 2 of [2]). If $f$ is a $\mathscr{U}^{N}$-invariant function on $\mathscr{H}^{N}$, then

$$
\int_{\mathscr{H}^{N}} f(H) d M(H)=\frac{\Omega_{N}}{(2 \pi)^{N}} \int_{\mathbf{R}^{N}} \prod_{1 \leqslant i<j \leqslant N}\left(\lambda_{i}-\lambda_{j}\right)^{2} f(\Lambda) \prod_{n=1}^{N} d \lambda_{n},
$$

where $\Lambda=\left(\Lambda_{i j}\right)$ is the diagonal matrix with $\Lambda_{i j}=\lambda_{i} \delta_{i j}, i, j=1, \cdots, N, d \lambda_{n}$ denotes Lebesgue measure, and $\Omega_{N}=2^{N} \pi^{N(N+1) / 2} / \Pi_{p=1}^{N} p$ !.

The factor $\Pi_{i \neq j}\left(\lambda_{i}-\lambda_{j}\right)$ which one picks up from the Jacobian here is sometimes called the "Weil factor." Integrating even very simple functions against this Weil factor is a substantial task; our next result describes a special case of such a computation.

As a prefatory note, we recall (see [7], for instance) that if $\mu$ is a measure defined on $\mathbf{R}$, then the "monic orthogonal polynomials for $\mu$ " are the polynomials $k_{n}(x)=x^{n}+\cdots$ with $\int_{\mathbf{R}} k_{n}(x) k_{m} d \mu(x)=0$ for $n \neq m$. Such polynomials exist and are unique provided the moments $\int_{\mathbf{R}} x^{n} d \mu(x)$ exist and are finite for $n=0,1, \cdots$. A simple inductive argument then shows that the coefficient of $k_{i}(x)$ in $x k_{n}(x)$ vanishes for $i \leqslant n-2$, so the orthogonal polynomials for $\mu$ satisfy a three-term recursion

$$
k_{n+1}(x)=\left(x+\alpha_{n}\right) k_{n}(x)-\rho_{n} k_{n-1}(x)
$$

for certain scalars $\alpha_{n}, \rho_{n}, n \geqslant 0$ (with $k_{-1}=0$ ).

Lemma 3.3 (see [2, pp. 123-124] for instance). Suppose $\mu$ is a measure on $R$ whose moments $\bar{\mu}_{n}=\int_{\mathbf{R}} x^{n} d \mu(x)$ exist and are finite for $n \geqslant 0$. If the three-term recursion for the monic orthogonal polynomials of $\mu$ is as above, then

$$
\int_{\mathbf{R}^{N}} \prod_{i \neq j}\left(x_{i}-x_{j}\right) \prod_{i=1}^{N} d \mu\left(x_{i}\right)=N !\left[\bar{\mu}_{0}\right]^{N} \prod_{n=1}^{N-1} \rho_{n}^{N-n} \text {. }
$$


Proof. Expanding the square root of the Weil factor as a Vandermonde determinant, the integral in the lemma admits the expression

$$
N ! \operatorname{det}\left(\int_{\mathbf{R}} p_{n-1}(x) p_{m-1}(x)\right)_{m, n=1, \cdots, N}
$$

for any family $\left\{p_{n}\right\}_{n \geqslant 0}$ of monic polynomials with degree $p_{n}=n$; indeed, for $p(x)=x^{n}$ this is just the Vandermonde determinant, and the general result follows by linearity. For $p_{n}=k_{n}$, the determinant computation is diagonalized, and we find

$$
\begin{aligned}
\int_{\mathbf{R}} k_{n+1}^{2}(x) d \mu(x) & =\int_{\mathbf{R}} k_{n+1}(x) x k_{n}(x) d \mu(x) \\
& =\int_{\mathbf{R}} d \mu(x) k_{n}(x)\left[k_{n+2}(x)-\alpha_{n+1} k_{n+1}(x)+\rho_{n+1} k_{n}(x)\right] \\
& =\rho_{n+1} \int_{\mathbf{R}} k_{n}^{2}(x) d \mu(x) . \quad \text { q.e.d. }
\end{aligned}
$$

In particular, with $d \mu(x)=e^{-x^{2} / 2} d x$ we find the monic Hermite polynomials $h_{n}$ (see [7, pp. $\left.101 \mathrm{ff}.\right]$ ) with recursion $h_{n+1}=x h_{n}-n h_{n-1}$ and $\bar{\mu}_{0}=\sqrt{2 \pi}$, so $\Phi(0, N)=1$. Lemma 3.3 will prove to be our main computational tool once the problem is appropriately set up.

Corollary 3.1 and Lemma 3.2 give

$$
\Phi(I, N)=\nu_{N} \sum_{\Sigma(k-2) v_{k}=I} \frac{1}{\prod_{k} v_{k} !} \int_{\mathbf{R}^{N}} \prod_{k}\left[-\frac{p_{k}}{k}\right]^{v_{k}} d L,
$$

where $p_{k}=\sum_{i=1}^{N} x_{i}^{k}$

$$
d L=\prod_{i \neq j}\left(x_{i}-x_{j}\right) e^{-p_{2} / 2} \prod_{i=1}^{N} d x_{i}
$$

and

$$
\nu_{N}=\frac{\Omega_{N}}{\mu_{N}(2 \pi)^{N}}=\frac{1}{(2 \pi)^{N / 2} \Pi_{p=1}^{N} p !} .
$$

Now we reindex, replacing the (ordered) tuple $v_{3}, \cdots, v_{K}$ with the (unordered) multi-index

$$
\lambda=\underbrace{1 \cdots 1}_{v_{3}}, \underbrace{2 \cdots 2}_{v_{4}}, \cdots, \underbrace{K-2 \cdots K-2}_{v_{K}},
$$


so that $|\lambda|=\sum_{k}(k-2) v_{k}$ and $v_{k}=m_{k-2}=\#\{(k-2)$ 's in $\lambda\}$. Thus, we have

$$
\Phi(I, N)=\nu_{N} \sum_{|\lambda|=I} \int_{\mathbf{R}^{N}} H_{\lambda} d L
$$

where

$$
H_{\lambda}=\prod_{k} \frac{1}{m_{k} !}\left[\frac{-p_{k+2}}{k+2}\right]^{m_{k}}
$$

and we write " $|\lambda|=I$ " to mean the sum over all multi-indices $\lambda$ with $|\lambda|=I$.

We seek the generating function

$$
Z(t, N)=\sum_{I \geqslant 0} \Phi(I, N)(i \sqrt{t})^{I},
$$

but (as we will see) it does not converge. Instead, we truncate spatially and take

$$
Z_{M}(t, N)=\sum_{I \geqslant 0} \Phi_{M}(I, N)(i \sqrt{t})^{I}, \quad t \geqslant 0,
$$

where $\Phi_{M}(I, N)=\nu_{N} \sum_{|\lambda|=I} \int_{B_{M}^{N}} H_{\lambda} d L$ and $B_{M}^{N}=\left\{x \in \mathbf{R}^{N}:|x| \leqslant M\right\}$. As before, $\Phi_{M}(I, N)=0$ for $I$ odd, so $Z_{M}(t, N)$ is real-valued and single-valued wherever it converges.

Lemma 3.4. $Z_{M}(t, N)$ converges absolutely for $0 \leqslant t<M^{-4}(e N)^{-2}$.

Proof. For fixed $\lambda$, on $B_{M}^{N}$ we have the estimate

$$
\left(\prod_{k} m_{k} !\right)\left|H_{\lambda}\right| \leqslant \prod_{k}\left|N M^{k+2}\right|^{m_{k}} \leqslant N^{I} M^{2 I} .
$$

Furthermore, if $\sum_{j} y_{j}$ converges absolutely, then

$$
\sum_{\lambda} \prod_{j} \frac{y^{m_{j}}}{m_{j} !}=\exp \left(\sum_{j} y_{j}\right),
$$

so in particular $\sum_{\lambda} \Pi_{k}\left(m_{k} !\right)^{-1}<e^{I}$. Thus,

$$
\begin{aligned}
\left|\nu_{N} \sum_{|\lambda|=I} \int_{B_{M}^{N}} H_{\lambda} d L(i \sqrt{t})^{I}\right| & \leqslant \nu_{N} \int_{B_{M}^{N}} d L N^{I} M^{2 I} e^{I} t^{I / 2} \\
& \leqslant \nu_{N} \int_{\mathbf{R}^{N}} d L N^{I} M^{2 I} e^{I} t^{I / 2}=N^{I} M^{2 I} e^{I} t^{I / 2}
\end{aligned}
$$

and the claim follows by the root rest. q.e.d. 
Now, we find

$$
\begin{aligned}
Z_{M}(t, N) & =\nu_{N} \sum_{I \geqslant 0}(i \sqrt{t})^{I} \sum_{|\lambda|=I} \int_{B_{M}^{N}} H_{\lambda} d L \\
& =\nu_{N} \int_{B_{M}^{N}} \sum_{\lambda}(i \sqrt{t})^{|\lambda|} H_{\lambda} d L \\
& =\nu_{N} \int_{B_{M}^{N}} \sum_{\lambda} \prod_{k}\left[\frac{-p_{k+2}(i \sqrt{t})^{k}}{k+2}\right]^{m_{k}} \frac{d L}{m_{k} !} \\
& =\nu_{N} \int_{B_{M}^{N}} \exp \left(-\left[\frac{p_{2}}{2}+\sum_{k} \frac{p_{k+2}(i \sqrt{t})^{k}}{k+2}\right]\right) \prod_{i \neq j}\left(x_{j}-x_{j}\right) \prod_{i=1}^{N} d x_{i} \\
& =\nu_{N} \int_{B_{M}^{N}} \prod_{i \neq j}\left(x_{i}-x_{j}\right) \prod_{i=1}^{N} d \mu_{t}\left(x_{i}\right)
\end{aligned}
$$

using Lemma 3.4 and another application of $(\dagger)$, where

$$
\begin{aligned}
d \mu_{t}(x) & =\exp \left[-\sum_{k \geqslant 0} \frac{x^{k+2}(i \sqrt{t})^{k}}{k+2}\right] d x \\
& =\exp \left[\frac{1}{t} \sum_{n=2}^{\infty} \frac{(i x \sqrt{t})^{n}}{n}\right] d x \\
& =\exp \frac{1}{t}\left[\log \frac{1}{1-i x \sqrt{t}}-i x \sqrt{t}\right] d x \\
& =(1-i x \sqrt{t})^{-t^{-1}} e^{-i x / \sqrt{t}} d x \\
& =-i \sqrt{t}(e t)^{-t^{-1}}(-z)^{-t^{-1}} e^{-z} d z, \text { for } z=\frac{1}{t}(i x \sqrt{t}-1)
\end{aligned}
$$

Now, let us take the limit of the functions $Z_{M}(t, N)$ which are analytic in a shrinking neighborhood of zero:

$$
\begin{aligned}
Z(t, N) & =\lim _{M \rightarrow \infty} Z_{M}(t, N) \\
& =\nu_{N} \int_{\mathbf{R}^{N}} \prod_{i \neq j}\left(x_{i}-x_{j}\right) \prod_{i=1}^{N} d \mu_{t}\left(x_{i}\right),
\end{aligned}
$$

where the integral is improper in the sense above. We wish to apply Lemma 3.3 and must find the orthogonal polynomials for $\mu_{t}(x), t>0$, on $\mathbf{R}$. To this end, 
we have

Lemma 3.5. If $q(x)$ is analytic and $t>0$, then

$$
\int_{\mathbf{R}} q((i x \sqrt{t}-1) / t) d \mu_{t}(x)=\frac{2 \pi \sqrt{t}(e t)^{-t^{-1}}}{\Gamma\left(1-t^{-1}\right) \Gamma\left(t^{-1}\right)} \int_{0}^{\infty} q(z) z^{-t^{-1}} e^{-z} d z .
$$

Proof (see Hankel's description of the gamma function [8, pp. 244-246]).

$$
\begin{aligned}
\int_{\mathbf{R}} q((i x \sqrt{t}-1) / t) d \mu_{t}(x) & =-i \sqrt{t}(e t)^{-t^{-1}} \int_{\operatorname{Re} z=-t^{-1}} q(z)(-z)^{-t^{-1}} e^{-z} d z \\
& =2 \sqrt{t}(e t)^{-t^{-1}} \sin \left(1-t^{-1}\right) \int_{\mathbf{R}^{+}} q(z) z^{-t^{-1}} e^{-z} d z \\
& =\frac{2 \pi \sqrt{t}(e t)^{-t^{-1}}}{\Gamma\left(1-t^{-1}\right) \Gamma\left(t^{-1}\right)} \int_{0}^{\infty} q(z) z^{-t^{-1}} e^{-z} d z
\end{aligned}
$$

Corollary 3.6. If $\bar{\mu}_{0}(t)$ denotes the zeroeth moment of the measure $\mu_{t}$ for $t>0$, then

$$
\bar{\mu}_{0}(t)=2 \pi \sqrt{t}(e t)^{-t^{-1}} \frac{1}{\Gamma\left(t^{-1}\right)} .
$$

We define a collection of polynomials for $t>0$,

$$
k_{n, t}(x)=\frac{t^{n / 2}}{i^{n}} l_{n}^{-t^{-1}}\left(i x / \sqrt{t}-t^{-1}\right)
$$

where $l_{n}^{\alpha}(y)$ is the $n$th monic Laguerre polynomial, which is orthogonal for the measure $y^{\alpha} e^{-y} d y$. We have

Corollary 3.7. $\left\{k_{n, t}\right\}$ is a collection of monic polynomials, which are orthogonal for $d \mu_{t}, t>0$, and satisfy the recursion relation

$$
k_{n+1, t}(x)=\{x+i \sqrt{t}(2 n+1)\} k_{n, t}(x)+n(t n-1) k_{n-1, t}(x) .
$$

Proof. The usual Laguerre polynomials $L_{n}^{\alpha}(z)$ (see pp. 96-97 of [7]) are normalized by $\int_{0}^{\infty} e^{-z} z^{\alpha} L_{n}^{\alpha} L_{m}^{\alpha} d z=\Gamma(\alpha+1)\left(\begin{array}{c}n+\alpha \\ n\end{array}\right) \delta_{n m}$ and the coefficient of $z^{n}$ in $L_{n}^{\alpha}(z)$ has sign $(-1)^{n}$. The $L_{n}^{\alpha}(z)$ satisfy a recursion

$$
(n+1) L_{n+1}^{\alpha}(z)=(-z+2 n+\alpha+1) L_{n}^{\alpha}(z)-(n+\alpha) L_{n-1}^{\alpha}(z),
$$

and $L_{n}^{\alpha}(z)=(-1)^{n} z^{n} / n !+O\left(z^{n-1}\right)$. Thus, $l_{n}^{\alpha}(z)$ has recursion

$$
l_{n+1}^{\alpha}(z)=(z-2 n-\alpha-1) l_{n}^{\alpha}(z)-n(n+\alpha) l_{n-1}^{\alpha}(z),
$$

so the $k_{n, t}(x)$ have the asserted recursion, are monic by construction, and orthogonal for $d \mu_{t}(x)$ by Lemma 3.5.

Theorem 3.8. As a formal power series, $Z(t, N)$ agrees with the asymptotic series at 0 of

$$
\left[\frac{\sqrt{2 \pi t}(e t)^{-t^{-1}}}{\Gamma\left(t^{-1}\right)}\right]_{p=1}^{N}(1-p t)^{N-p}
$$


Proof. Apply Lemma 3.3 to the measure $\mu_{t}$ for $t>0$ using Corollaries 3.6 and 3.7. q.e.d.

We relate $\log Z(t, N)$ to the generating function $\Phi_{c}(I, N)$ (introduced in $\S 1$ ) for connected fat graphs. If $G$ is a fat graph of components $G=$ $\amalg_{c=1}^{C}\left(a_{c}\right.$ copies of $\left.G_{c}\right)$, then

$$
\begin{gathered}
\# \Gamma[G]=\prod_{c=1}^{C} a_{c} !\left(\# \Gamma\left[G_{c}\right]\right)^{a_{c}}, \\
N^{s[G]}=\prod_{c=1}^{C} N^{a_{c} s\left[G_{c}\right]} .
\end{gathered}
$$

A standard generating function analysis (see [2, p. 115] for instance) then gives the following formal equality:

$$
-\log Z(t, N)=\sum_{I \geqslant 1} \Phi_{c}(2 I, N)(-t)^{I}
$$

We have arrived at the main result of the paper.

Theorem 3.9. Fix $s \geqslant 1, g \geqslant 0$ with $2 g-2+s>0$. We have

$$
\chi_{v} \mathrm{MC}_{g}^{s}=\sum_{\begin{array}{c}
\text { connected }[G] \\
\text { with } s[G]=s \\
g[G]=g
\end{array}} \frac{(-1)^{V[G]}}{\# \Gamma[G]}=(-1)^{s} \frac{(s+2 g-3) !(2 g-1)}{s !(2 g) !} B_{2 g} .
$$

Proof. Taking the logarithm of the expression in Theorem 3.8, invoking Stirling's formula, and expanding $\log (1-p t)$ in a power series, we immediately get

$$
\log Z(t, N)=\sum_{j=1}^{\infty} t^{j}\left[-N \frac{B_{j+1}}{j(j+1)}-\frac{1}{j} \sum_{p=1}^{N-1}(N-p) p^{j}\right] .
$$

Using the standard formula

$$
\sum_{p=1}^{N-1} p^{v}=\frac{N^{v+1}}{v+1}-\frac{N^{v}}{2}+\sum_{k=1}^{[v / 2]}\left(\begin{array}{c}
v \\
2 k-1
\end{array}\right) \frac{B_{2 k}}{2 k} N^{v+1-2 k},
$$

it follows that

$$
\begin{aligned}
\log Z(t, N)=\sum_{j=1}^{\infty} t^{j}[ & -\frac{N^{j+2}}{j(j+1)(j+2)} \\
& \left.+\sum_{k=1}^{[(j+1) / 2]}(2 k-1) \frac{(j-1) !}{(j+2-2 k) !} \frac{B_{2 k}}{(2 k) !} N^{j+2-2 k}\right],
\end{aligned}
$$

proving the theorem. 
Corollary 3.10. If $\mathrm{MC}_{g}^{0}$ denotes the mapping class group of the genus $g$ surface, $g>1$, with no punctures, then

$$
\chi_{v} \mathrm{MC}_{g}^{0}=\frac{B_{2 g}}{4 g(g-1)} \text {. }
$$

Proof. Suppose $* \rightarrow A \rightarrow B \rightarrow C \rightarrow *$ is a short exact sequence of groups, where $B$ is virtually torsion free and $A, C$ satisfy condition VFP of [4] ( $A$ satisfies VFP if it contains a finite index subgroup $D$ so that the trivial $\mathbf{Z}[D]$-module $\mathbf{Z}$ admits a finite resolution by finitely generated projective $\mathrm{Z}[D]$-modules); Corollary 2 to Theorem 4 of [4] gives $\chi_{v} B=\left(\chi_{v} A\right)\left(\chi_{v} C\right)$. Applying this observation to the short exact sequence $* \rightarrow \pi_{1} F_{g}^{s} \rightarrow \mathrm{MC}_{g}^{1} \rightarrow$ $\mathrm{MC}_{g}^{0} \rightarrow *$ yields the result.

Remarks. (1) The behavior of virtual Euler characteristic with respect to short exact sequences described above allows a consistency check on our computations. Namely, $\chi_{v} \mathrm{MC}_{g}^{s}, s \neq 1$, can be derived from $\chi_{v} \mathrm{MC}_{g}^{1}$ using various short exact sequences; the results so obtained are in agreement with Theorem 3.9.

(2) We mention that our formula for $\chi_{v} \mathrm{MC}_{g}^{s}, s>1$, differs from that in [5] by the factor $1 / s$ !. This is because [5] considers not the full mapping class group, but the subgroup of mapping classes which fix the punctures.

We close by mentioning several related open problems. It would perhaps be interesting to compute the "partition function" $Z(t, N)$ for classical groups other than $\mathscr{H}^{N}$. The techniques in this paper should also apply to the computation of an analogous generating function for orders of symmetry groups of graphs (not fat graphs). Furthermore, one would like to know the number of cells of various codimensions in the cell decomposition of $\tilde{\mathscr{T}}_{g}^{s}$, and it is possible that an extension of the method herein to the setting of non-Gaussian measures would yield such a generating function. Finally, the techniques of this paper together with the " $\lambda$-length" coordinatization of $\tilde{\mathscr{T}}_{g}^{s}$ in [6] give a reasonable description of a fundamental domain for the action of $\mathrm{MC}_{g}^{s}$ on $\tilde{\mathscr{T}}_{g}^{s}$. One should thus be able to integate over the moduli space using these ideas. In particular, the Weil-Petersson mass of moduli space seems a promising such computation.

\section{References}

[1] W. Abikoff, The real-analytic theory of Teichmüller space, Lecture Notes in Math., Vol. 820, Springer, New York, 1980.

[2] D. Bessis, C. Itzykson \& J. B. Zuber, Quantum field theory techniques in graphical enumeration, Advances in Appl. Math. 1 (1980) 109-157.

[3] J. Birman, Braids, links, and mapping class groups, Annals of Math. Studies, No. 82, Princeton University Press, Princeton, NJ, 1974. 
[4] K. S. Brown, Euler characteristics of discrete groups and G-spaces, Invent. Miath. 27 (1974) 229-264.

[5] J. Harer \& D. Zagier, The Euler characteristic of the moduli space of curves, Invent. Math. 85 (1986) 457-485.

[6] R. C. Penner, The Teichmüller space of a punctured surface, Comm. Math. Phys. (1987).

[7] G. Szego, Orthogonal polynomials, Amer. Math. Soc. Colloq. Publ. No. 23, Amer. Math. Soc., Providence, RI, 1939.

[8] E. T. Whittaker \& G. N. Watson, A course in modern analysis, Cambridge University Press, Cambridge, 1940.

University of SOUThern CALIForNia 
